\title{
Scaling of saturation and hue in the nonspectral region
}

\author{
TAROW INDOW \\ Keio University, Mita Minato-ku, Tokyo, Japan
}

\begin{abstract}
The saturation of two nonspectral hues, magenta and violet, and the hue shift between red and blue through the nonspectral region were both scaled by two methods, equisection to produce a difference scale, $\psi_{\mathrm{D}}$, and ratio judgments to produce a magnitude scale, $\psi_{\mathrm{M}}$. The colored stimuli were viewed through apertures in a dark surround, with the luminance kept constant at one of three levels, $0.5,2.5$, and $12.6 \mathrm{~cd} / \mathrm{m}^{2}$. As previously found with saturation and hue shift between two adjacent primary hues in the spectral region, $\psi_{D}$ was linear with colorimetric purity for the saturation of magenta or violet and with the mixture ratio of red or blue for the hue shift, whereas $\psi_{M}$ was a power function of colorimetric purity for the saturations and of the mixture ratio for the hue shift with exponents larger than unity in both cases. The present results were combined with previous results to give the change of parameters for saturation functions over the entire hue circle.
\end{abstract}

The present study concludes a series of experiments in which the saturation and hue shift of aperture colors in a dark surround were scaled on the basis of two kinds of judgment (Indow, 1967; Indow \& S. S. Stevens, 1966). In the present paper, a scale constructed by equisection is called a difference scale and denoted by $\psi_{\mathrm{D}}$ and a scale constructed by magnitude production and estimation is called a magnitude scale and denoted by $\psi_{\mathrm{M}}$. As has been demonstrated in other sensory modalities (S. S. Stevens, 1961, 1975), the two scales for saturation and hue shift are not linearly related and the magnitude scale, $\psi_{\mathrm{M}}$, is concave upward when plotted against the difference scale, $\psi_{\mathrm{D}}$ (Indow, 1974a, 1974b).

The following findings were reported in Indow and S. S. Stevens (1966). For saturation of each of the primary hues (red, yellow, green, and blue), when the luminance is kept constant, $\psi_{\mathrm{D}}$ is always a linear function and $\psi_{M}$ is always a power function of the colorimetric purity of the monochromatic stimulus. The same is true with saturation of the intermediate hues, orange and yellowish green. In addition, mixing monochromatic stimuli of the two adjacent primary hues, red and yellow or yellow and green, behaves much the same as mixing a primary hue with achromatic light. Of a hue component in the resulting hue shift, e.g., redness in orange, $\psi_{\mathrm{D}}$ is a linear function and $\psi_{\mathrm{M}}$ is a power function

The experiments reported in this paper were carried out in June 1972, in the Harvard University Laboratory of Psychophysics directed by the late Professor S. S. Stevens. The computations were made after the author returned to Tokyo. Professor R. Mansfield of Harvard and Mrs. S. S. Stevens kindly read through the first draft and gave valuable suggestions concerning the style of English. The final draft was completed while the author was a visiting professor at the University of California, Irvine. of the mixture ratio of the hue. In the present study, the same type of scaling is extended to saturation of two nonspectral hues, magenta and violet, and to the hue shift in the nonspectral region from red to blue.

The experiments are referred to by the numbers given in Table 1. The hues are denoted by the alphabetic abbreviations: red, R; orange, OR; yellow, Y; yellowish green, GY; green, G; blue, B; violet, V; and magenta, $M$. As in the previous articles, luminance is expressed in decibels re $10^{-10} \mathrm{~L}$, which corresponds approximately to the absolute threshold of the darkadapted eye (S. S. Stevens, 1955). A level of $1 \mathrm{~cd} / \mathrm{m}^{2}$ corresponds to $65 \mathrm{~dB}$.

\section{GENERAL METHOD}

\section{Apparatus}

The structure of the apparatus was described in detail in Indow and S. S. Stevens (1966), and a brief description will suffice. By means of a rotating prism, lights were mixed and presented through five apertures arranged in a row: Aperture 1 to Aperture 5 from left to right. In each aperture, the mixing ratio of two lights was varied continuously and independently, keeping the luminance at a constant level. The subject monocularly observed one ar more apertures, depending upon the experiment, through a rectangular slit, $3 \mathrm{~mm}$ high and $15 \mathrm{~mm}$ wide, at a distance of $79 \mathrm{~cm}$. Each aperture was about $30^{\prime}$ long and $22^{\prime}$ high in visual angle, and the subtense of the five apertures in a row was $9^{\circ}$. Between presentations, the eye was exposed to a gray covering the row of apertures and the gray was illuminated by a beam of light of about $6,500^{\circ} \mathrm{K}(65 \mathrm{~dB})$. When one or more apertures were uncovered, this beam of light was shunted. The other beam from the same light source evenly illuminated from below a row of five microscope glass slides, one under each aperture. On each glass slide, pairs of contiguous filters were mounted and the subject could move each slide smoothly and continuously at any speed and accuracy, by which the mixing ratio of lights coming through the pair of filters on each glass slide was changed. When the saturation of a hue was investigated, a monochromatic filter and neutral density filter were paired, and when the hue shift between $R$ and $B$ was 
Table 1

List of Experiments

\begin{tabular}{|c|c|c|c|c|}
\hline $\begin{array}{c}\text { Experi- } \\
\text { ment }\end{array}$ & Description & $\begin{array}{c}\text { Number } \\
\text { of Stimuli }\end{array}$ & $\mathbf{N}$ & $\begin{array}{l}\text { Repe- } \\
\text { titions }\end{array}$ \\
\hline Series 1 & Saturation of four hues; $R, M, V$, and $B$ at three levels of luminance $(62,69,76 \mathrm{~dB})$ & & & \\
\hline 1.1 & Equisection ( $M$ and $V$ at $69 \mathrm{~dB}$ only) & 5 & 10 & $2 \times 2 *$ \\
\hline 1.2 & Absolute threshold (method of limits, ascending) & 1 & 7 & 2 \\
\hline 1.3 & Magnitude production and estimation & $5-20$ & 15 & 1 \\
\hline 1.4 & Heterochromatic matching & 5 & 11 & 4-7 \\
\hline Series 2 & Hue shift between $R$ and $B$ at three levels of luminance $(62,69,76 \mathrm{~dB})$ & & & \\
\hline 2.1 & Equisection & 5 & 15 & $2 \times 2 *$ \\
\hline 2.2 & Absolute threshold (method of limits) & 1 & 15 & 2 \\
\hline 2.3 & Matching of $M$ and $V(M a x . P c)$ by mixing $R$ and $B$ & 4 & 15 & 2 \\
\hline 2.4 & Magnitude estimation of component hue & 6 & 15 & $2^{*}$ \\
\hline 2.5 & Magnitude production and estimation of a component hue & $9-25$ & 15 & 2* \\
\hline 2.6 & Matching between saturation and a component hue & 3,5 & 10 & 2 \\
\hline
\end{tabular}

Note-Number of stimuli refers to the number of stimuli presented at one time or used in a series. $N$ refers to the number of subjects. *Entire series repeated twice.

investigated, two monochromatic filters of $\mathrm{R}$ and $\mathrm{B}$ were paired. The filters to be paired were always equated in luminous transparency. The mixing ratio, $\phi$, is denoted by the colorimetric purity, $\mathrm{Pc}$, for saturation and by the percentage of the $\mathrm{R}$ component for the hue shift between $R$ and $B$, unless otherwise stated.

Wratten chromatic gelatin filters used are given in Table 2. Wratten neutral density filters No. 96, of various attenuation factors, were combined with the chromatic filters so as to equate the luminous transparencies of the two parts on each glass slide and also to set the stimuli at one of the following luminance levels: 62,69 , or $76 \mathrm{~dB}$.

\section{Subjects}

Except for the requirement of normal color vision, thirteen subjects were selected in a random fashion from among the graduate students in psychology. They had neither technical knowledge of color nor experience in serving as subjects in an experiment of this kind. Two additional subjects were the author and one other. The latter two had participated in the previous experiments. The experiments were carried out individually in the order given in Table 1, and five sessions were required for a subject to complete all the experiments, each session being less than $50 \mathrm{~min}$. Some experiments could not be run with some subjects because of the time limits; the number of subjects, $\mathrm{N}$, in each experiment is given in Table 1 .

\section{Procedure}

Using his preferred eye, the subject adapted the eye to the gray field $(65 \mathrm{~dB})$ for 2 min at the beginning of each experiment. As soon as the light illuminating the adapting field was turned off, one stimulus or a row of stimuli were presented in the dark surround. Apertures not in use were invisible. When more than one stimulus was presented, the subject was encouraged to move his eye to fixate them one by one. The procedure of each experiment is described below.

\section{SATURATION OF FOUR HUES}

In the first series of experiments, the saturation of four hues, red $(R)$, magenta $(M)$, violet $(V)$, and blue $(B)$, were scaled. Hence, $\phi$ represents colorimetric purity, $\mathrm{Pc}$, of the hue under discussion, $\psi_{\mathrm{D}}$ and $\psi_{\mathrm{M}}$ represent, respectively, the difference and magnitude scales of the saturation.

\section{Experiments 1.1, 1.2}

Equisection was carried out in Experiment 1.1 for the two nonspectral hues, $\mathrm{M}$ and $\mathrm{B}$, at only one luminance level $(69 \mathrm{~dB})$.

The essential part of the instructions in the equisection of $M$ was as follows: "You will see a row of five colors. One end is gray and the other end is magenta. Your task is to adjust the three middle colors and to construct a series of colors that appear to mark off equal steps from one color to the next. In other words, the colors in the constructed series are to vary gradually from gray to magenta in equal steps. During the course of the adjustment, take care to look at each color one by one and to scan the whole series. You may start with either of the three adjustable colors. Try to work rapidly. Once the adjustments are made, you will be asked to look at a homogeneous surface for a while, and then the five colors will be presented again so that you may correct your adjustments if you like."

The equisection settings were made twice in each of the two spatial arrangements, $M$ of $100 \%$ once at the left end and once at the right end. The procedure was the same for the equisection of $\mathrm{V}$.

In Experiment 1.2, the absolute thresholds, $\phi_{0}$, for the four hues at three luminance levels were determined by the method of limits with ascending series for seven subjects. The stimulus was presented in the aperture in the center, and the subject was asked to approach the

Table 2

Characteristics of Chromatic Filters

\begin{tabular}{ccccc}
\hline Hue & $\begin{array}{c}\text { Wratten } \\
\text { Number }\end{array}$ & $\begin{array}{c}\text { Wave- } \\
\text { length* }\end{array}$ & $\begin{array}{c}\text { Excitation } \\
\text { Purity }\end{array}$ & $\begin{array}{c}\text { Attenu- } \\
\text { ation** }\end{array}$ \\
\hline R & 29 & 631.6 & 100.0 & 12.0 \\
B & 48 & 466.5 & 96.1 & 17.1 \\
M & 32 & $551.8 \mathrm{c}$ & 79.5 & 9.0 \\
V & $34 A$ & $565.6 \mathrm{c}$ & 90.6 & 15.4 \\
\hline
\end{tabular}

*Wavelength in $\lambda d$ in nanometers.

**Attenuation in decibels. 
threshold by increasing $\mathrm{Pc}$ from $0 \%$. The determination was repeated twice with an interval of $7 \mathrm{sec}$, during which the eye was exposed to the adapting field. The means of the two settings by the seven subjects are given in Figure 1, and, for comparison, the thresholds, $\phi_{0}$, for R and B obtained by the method of limits with ascending and descending series in the previous study (Indow \& S. S. Stevens, 1966, Experiment 2.3) are also included. The thresholds, $\phi_{0}$, obtained in the present experiment (unfilled circles and squares) were considerably higher in value than those obtained in the previous experiment (filled circles and squares), ${ }^{1}$ but both sets of results are the same in general form as a function of luminance in each hue. The thresholds for $M$ (crosses) are closer in pattern to those for $R$, and the thresholds for $\mathrm{V}$ (triangles) are closer in pattern to those for $B$, which accords with the position of $M$ and $V$ along the segment connecting $R$ and $B$.

Figure 2 shows the results of equisection where each point represents the mean of 40 settings: two repetitions in each of the two series by 10 subjects. The results were fitted by straight lines for saturation of $\mathrm{V}$ and $\mathrm{M}$, respectively, which conforms to the results obtained with other hues (Indow \& S.S. Stevens, 1966, Experiments 1.3, 4.3). The filled circles at both ends represent the standard stimuli, and the thresholds for $69 \mathrm{~dB}$ obtained in Experiment 1.2 are indicated by the arrows. As was the case in the previous study, the straight line intersects the horizontal axis between the origin and the threshold. The horizontal bars represent twice the interindividual standard deviations of settings for the 10 subjects. The standard deviation in the equisection tended to increase as a function of $\mathrm{Pc}$ inasmuch as the differential threshold of saturation was also an increasing function of $P c$ in each spectral color (Indow, 1967; Indow \& S. S. Stevens, 1966). In Figure 2, that tendency is clear for $\mathrm{V}$ but not so clear for $M$.

\section{Experiment 1.3}

In order to obtain individual magnitude scales of saturation, the method of combining magnitude produc-

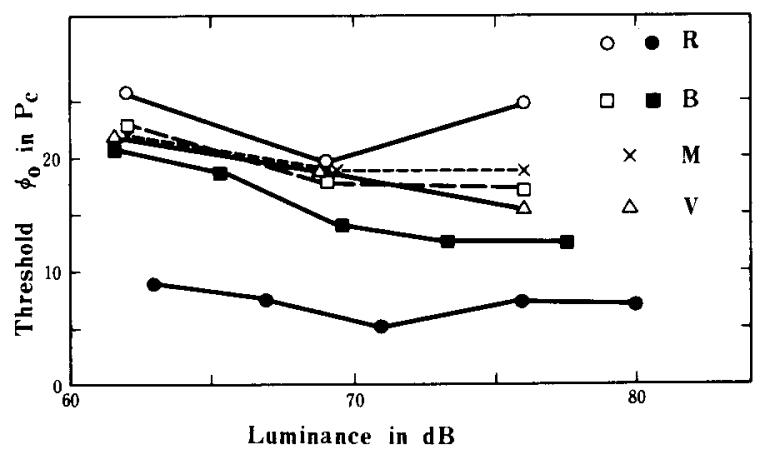

Figure 1. Absolute thresholds of saturation as a function of luminance. Unfilled marks represent results of Experiment 1.2; filled marks, results of a previous experiment (Indow \& S. S. Stevens, 1966).

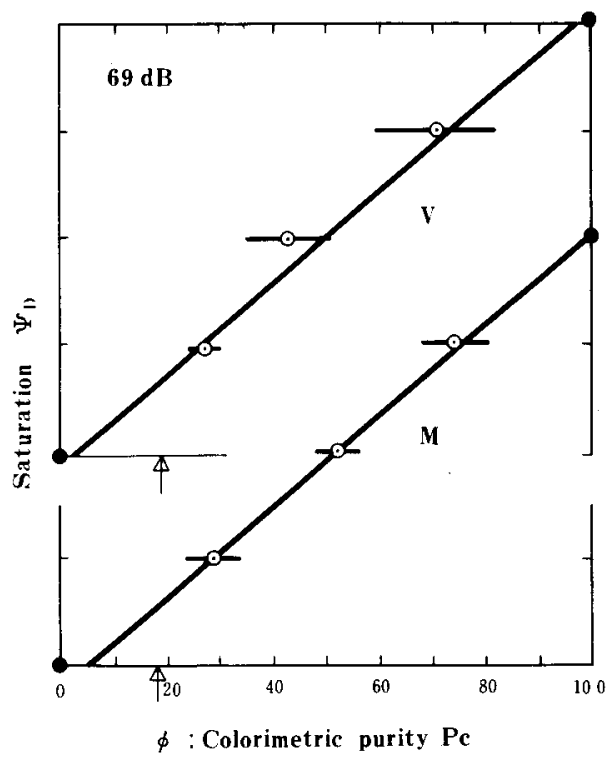

Figure 2. Equisections of saturation of magenta, $M$, and violet, $v$ (Experiment 1.1). Horizontal bars represent standard deviation of settings, arrows represent absolute thresholds of saturation (Experiment 1.2).

tion and estimation was used. A color was presented in the aperture at the center. The subject was asked to set the color to different levels of saturation and to assign numbers, $\psi_{M}$, to each level, $\phi$, in proportion to the saturation produced. The initial stimulus was randomly chosen in the middle range of $\mathrm{Pc}$, and the number assigned by the subject to this stimulus played the role of a modulus for the subject's scale, $\psi_{\mathrm{M}}$. Because the color remained in the subject's sight while its Pc was varied, the impression could be based both on the level, $\phi$, set by the subject and on the transient phase required to reach that level. The subject was encouraged to cover as wide a range of saturation as possible and to make settings and judgments quickly. When the whole range of $\mathrm{Pc}$ was covered with the appropriate number of settings (5 to 20), the series was terminated. This method was proved useful in giving results stable enough to define individual sensation functions (J.C. Stevens \& Guirao, 1964; Marks \& J. C. Stevens, 1966).

Six examples of individual saturation functions, $\psi_{M}(\phi)$, with three different levels of goodness of fit were shown in Indow (1974b, Figure 2). In each individual case, the numbers assigned, $\psi_{\mathrm{M}}(\phi)$, are approximated quite well by a power function. All the fittings were made by the method of least squares in which $\log \phi$ was taken as the independent variable. The statistics concerning 15 individual values of the exponent, $\beta$, and of the correlation, r, between $\psi_{\mathrm{M}}(\phi)$ and $\log \phi$ are given in Table 3. For the sake of comparison, the values of $\beta$ for $R$ and $B$ for the respective luminance levels that were estimated from Figure 11 of the previous article (Indow \& S. S. Stevens, 1966) are given in the last column. Those values were based mainly 
Table 3

Exponents and Goodness of Fit for Magnitude Production and Estimation of Saturation

\begin{tabular}{|c|c|c|c|c|c|c|}
\hline \multirow[b]{2}{*}{ Hue } & \multicolumn{4}{|c|}{ Exponent: $\beta$ in Experiment 1.3} & \multirow{2}{*}{$\begin{array}{c}\mathrm{r}^{* *} \\
\text { Range }\end{array}$} & \multirow{2}{*}{$\begin{array}{c}\text { Previous } \\
\beta \dagger\end{array}$} \\
\hline & Luminance* & Mean & SD & Range & & \\
\hline $\mathbf{R}$ & $\begin{array}{l}76 \\
69 \\
62\end{array}$ & $\begin{array}{l}2.90 \\
2.95 \\
2.17\end{array}$ & $\begin{array}{r}1.12 \\
.95 \\
.65\end{array}$ & $\begin{array}{l}1.39-6.20 \\
1.24-5.73 \\
1.06-3.39\end{array}$ & $\begin{array}{l}.79-.99 \\
.79-.99 \\
.74-.99\end{array}$ & $\begin{array}{l}1.73 \\
1.69 \\
1.65\end{array}$ \\
\hline B & $\begin{array}{l}76 \\
69 \\
62\end{array}$ & $\begin{array}{l}2.40 \\
2.06 \\
1.77\end{array}$ & $\begin{array}{r}1.17 \\
.76 \\
.98\end{array}$ & $\begin{array}{l}.80-4.77 \\
.86-4.46 \\
.44-3.21\end{array}$ & $\begin{array}{r}.74-.99 \\
.72-.99 \\
.74-.99\end{array}$ & $\begin{array}{l}1.65 \\
1.56 \\
1.48\end{array}$ \\
\hline $\mathbf{M}$ & $\begin{array}{l}76 \\
69 \\
62\end{array}$ & $\begin{array}{l}2.52 \\
2.31 \\
2.03\end{array}$ & $\begin{array}{r}1.14 \\
.97 \\
.72\end{array}$ & $\begin{array}{l}.33-5.28 \\
.99-3.59 \\
.47-3.44\end{array}$ & $\begin{array}{l}.23-.98 \\
.84-.98 \\
.81-.99\end{array}$ & \\
\hline V & $\begin{array}{l}76 \\
69 \\
62\end{array}$ & $\begin{array}{l}1.94 \\
1.63 \\
1.58\end{array}$ & $\begin{array}{l}.88 \\
.58 \\
.46\end{array}$ & $\begin{array}{l}.63-4.54 \\
.70-2.56 \\
.68-3.33\end{array}$ & $\begin{array}{l}.92-.99 \\
.84-.99 \\
.82-.99\end{array}$ & \\
\hline
\end{tabular}

${ }^{*} d B$ re $10^{-10} \mathrm{~L} . \quad{ }^{* *}$ Correlation between $\log \psi_{M}(\phi)$ and $\log \phi$.

tEstimated from Figure 11 of Indow and Stevens, 1966.

upon magnitude estimation as well as heterochromatic and heteroluminous matchings.

The exponents, $\beta$, obtained in Experiment 1.3 are larger in value than those of the previous study. Because the present procedure included magnitude production in which $\phi$ was set by the subject, what S. S. Stevens (1975) called the regression effect may have taken place. The effect tends to introduce regression of $\log \phi$ upon $\log \psi_{M}$ and hence to make $\psi_{M}(\phi)$ steeper in this case. Actually, overestimation of the exponent by the present procedure compared with the magnitude estimation alone has been reported for loudness (J. C. Stevens \& Guirao, 1964) but not for brightness (Marks \& J. C. Stevens, 1966). However, the overestimation of the exponent for loudness was of the order of magnitude of $1.2: 1$, whereas the overestimations of $\beta$ in the present case were greater, in general. The overestimation may also be ascribed to the visibility of the transient phase of the stimulus in the present experiment. It is plausible that observing the shift from one level to another magnifies the impression of that ratio between the two levels. In the above cited experiments on loudness and brightness, the transient phase of stimulus was not observable.

\section{Experiment 1.4}

Heterochromatic matches of saturation were made by 11 subjects among five colors, including two reds (two Rs, M, V, B), presented simultaneously in a row. The aperture at the left end was always occupied by $R$, and the remaining four colors were randomly presented in a row with different orders for different subjects, under the constraint that the two reds not be presented side by side. All five colors were of the same luminance, and three separate sets of matches were carried out for 76,69 , and $62 \mathrm{~dB}$.

The essential part of the instructions was as follows: "Among the five colors, one is considered to be a standard color and the remaining ones are adjustable colors. Your task is to adjust the adjustable colors to make them appear equal to the standard color in saturation. You may start with any one of the four and you should try to work rapidly. Once all the adjustments are completed, you will be asked to look at a homogeneous surface for a while, and then the five colors will be presented again so that you may correct your adjustments if you like."

One color randomly chosen from the five colors was designated as a standard and set by the experimenter at an appropriate level of Pc. In this way four to seven standard colors were used in a series so as to cover the widest possible range of $\mathrm{Pc}$ and also not to "scale out" any of the four adjustable colors.

Let us take two colors, $\mathrm{j}$ and $\mathrm{k}$, from the five colors thus matched. If saturations $\psi_{j}$ and $\psi_{k}$ are given in the same unit and

$$
\psi_{\mathrm{j}}=\alpha_{\mathrm{j}} \phi^{\beta_{\mathrm{j}}}, \quad \psi_{\mathrm{k}}=\alpha_{\mathrm{k}} \phi^{\beta_{\mathrm{k}}}
$$

hold, then we have

$$
\begin{gathered}
\log \phi_{j}=\log a_{j k}+b_{j k} \log \phi_{k} \\
a_{j k}={\frac{\alpha_{k}}{\alpha_{j}}}^{\frac{1}{\beta_{j}}} \quad b_{j k}=\frac{\beta_{k}}{\beta_{j}} .
\end{gathered}
$$

As was found in the previous study (Indow \& S. S. Stevens, 1966), four to seven settings of $\phi_{\mathrm{j}}$ and $\phi_{\mathrm{k}}$ were linearly scattered in log-log coordinates for every pair of $j$ and $k$ in each subject. The linearity can be described in terms of the correlation coefficient, $r$, in $\log -\log$ coordinates, and the interquartile ranges of $\mathrm{r}$ over 11 subjects and 18 pairs were $0.83-0.96$ for $76 \mathrm{~dB}$, 0.96-0.93 for $69 \mathrm{~dB}$, and $0.66-0.87$ for $62 \mathrm{~dB}$. The parameters, $\log a_{j k \cdot i}$ and $b_{j k \cdot i}(i=1,2, \cdots, 11)$, were individually estimated by the double regression method 
(Indow \& S. S. Stevens, 1966). Then respective averages of these 11 values were defined as $\log a_{j k}$ and $\log b_{j k}$. From those values were obtained the solutions of $\log \alpha_{j}$ and $\log \beta_{\mathrm{j}}$ in the least-squares sense (Mosteller, 1951):

$$
\begin{aligned}
& \log \alpha_{\mathrm{j}}^{\prime}=\frac{\beta_{\mathrm{j}}}{5} \sum_{\mathrm{k}} \log \mathrm{a}_{\mathrm{jk}}, \\
& \log \beta_{\mathrm{j}}^{\prime}=\frac{1}{5} \sum_{\mathrm{k}} \log b_{\mathrm{jk}} .
\end{aligned}
$$

Because the values of $\alpha_{j}^{\prime}$ and $\beta_{j}^{\prime}$ thus obtained $(j=1,2$, $\cdots, 5)$ are defined with the respective units such that

$$
\prod_{\mathrm{j}} \alpha_{\mathrm{j}}^{\prime}=1, \quad \prod_{\mathrm{j}} \beta_{\mathrm{j}}^{\prime}=1,
$$

these $\alpha_{\mathrm{j}}^{\prime}$ and $\beta_{\mathrm{j}}^{\prime}$ will be converted later to $\alpha_{\mathrm{j}}$ and $\beta_{\mathrm{j}}$, which have the appropriate units in the sense to be described later. Because there were two reds, two values were obtained for $\alpha$ and $\beta$, respectively, and hence the mean values were adopted as $\alpha$ and $\beta$ of $R$.

Consistency in heterochromatic matchings can be checked in the following way. In theory, each of the following plots should exhibit a scatter of points along the straight line passing through the origin with unit slope:

$$
\begin{aligned}
& \log a_{j k} \text { vs. } \frac{1}{\beta_{j}}\left(\log \alpha_{k}^{\prime}-\log \alpha_{j}^{\prime}\right) \\
& \log b_{j k} \text { vs. }\left(\log \beta_{k}^{\prime}-\log \beta_{j}^{\prime}\right) .
\end{aligned}
$$

The results are shown in Figure 3. The lower plot guarantees only the consistency in proportionality between relative values of $\beta_{\mathrm{j}}$ and $\beta_{\mathrm{k}}$, and in order to obtain $\alpha_{j}^{\prime}$ and the upper plot, an absolute value of one $\beta_{0}$ has to be given. For reasons to be stated later, the values of $\beta$ for $R$, as shown in Figure 3, were used as $\beta_{0}$. Though slight, a systematic bias is observable in the upper plot but not in the lower. Values of $\alpha$ and $\beta$ finally obtained in this experiment are given in Figure 6 and will be discussed in the last section.

\section{HUE SHIFT BETWEEN R AND B}

In this series of experiments, the mixture ratio of the $\mathrm{R}$ component is denoted by $\phi$, unless otherwise stated, and $\psi_{\mathrm{D}}$ or $\psi_{\mathrm{M}}$ represents the difference scale or the magnitude scale of either redness or blueness in a color.

\section{Experiments 2.1, 2.2, 2.3}

In Experiment 2.1, equisection was carried out for 15 subjects exactly as described in Experiment 1.1. For example, equisection settings were made twice

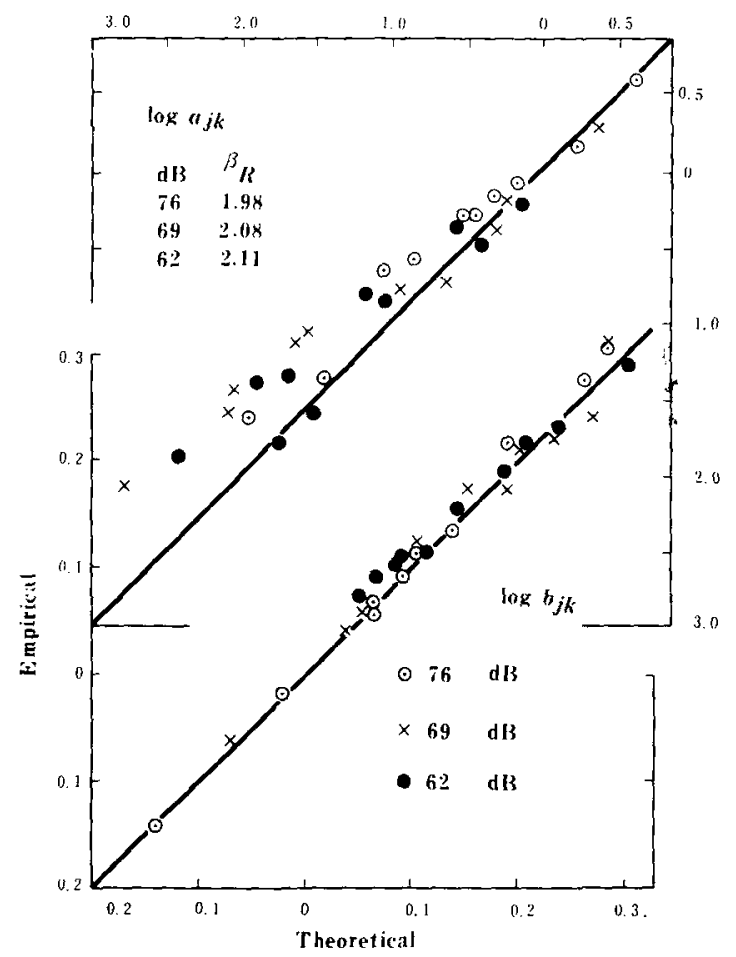

Figure 3. Consistencies of heterochromatic matching of saturation (Experiment 1.4). Abscissa represents $\log \beta_{k}^{\prime} / \beta^{\prime}{ }_{j}$ or $1 / \beta \log \alpha_{k}^{\prime} / \alpha^{\prime}{ }_{j}$ of Equation 3, ordinate $\log b_{j k}$ or $\log a_{j k}$ in matching colors $j$ and $k$ (see text).

in the arrangement of $R$ at the left end and $B$ at the right end and twice in the reverse arrangement. The unfilled circles in Figure 4 represent means of 60 settings each (two repetitions in the two arrangements by 15 subjects) and are approximated very closely by the straight line directly connecting the two standard stimuli (filled circles) at each luminance level. The same was true for hue shifts between $R$ and $Y$, as well as those between $Y$ and $G$ (Indow \& S. S. Stevens, 1966, Figure 16). As in Figure 2, horizontal bars represent twice the interindividual standard deviations. Unlike the equisections of saturation, the standard deviations tend to be largest in the middle rather than at either end.

The arrows on each side of the abscissa represent the absolute threshold by the method of limits in Experiment 2.2. Arrows pointing up represent the thresholds by ascending series and arrows pointing down represent those by the descending series. Each arrow is the mean of 30 settings (two repetitions by the 15 subjects). From the specifications of the filters, No. 32 and No. 34A (Table 2), $M$ and $V$ can be located along the continuum, $\phi$, at the points shown by the filled triangles in Figure 4. In Experiment 2.3, the value $\phi$ was determined by the method of adjustment that yields the color perceptually closest to either $M$ or V. A standard color, either M or V, was presented 


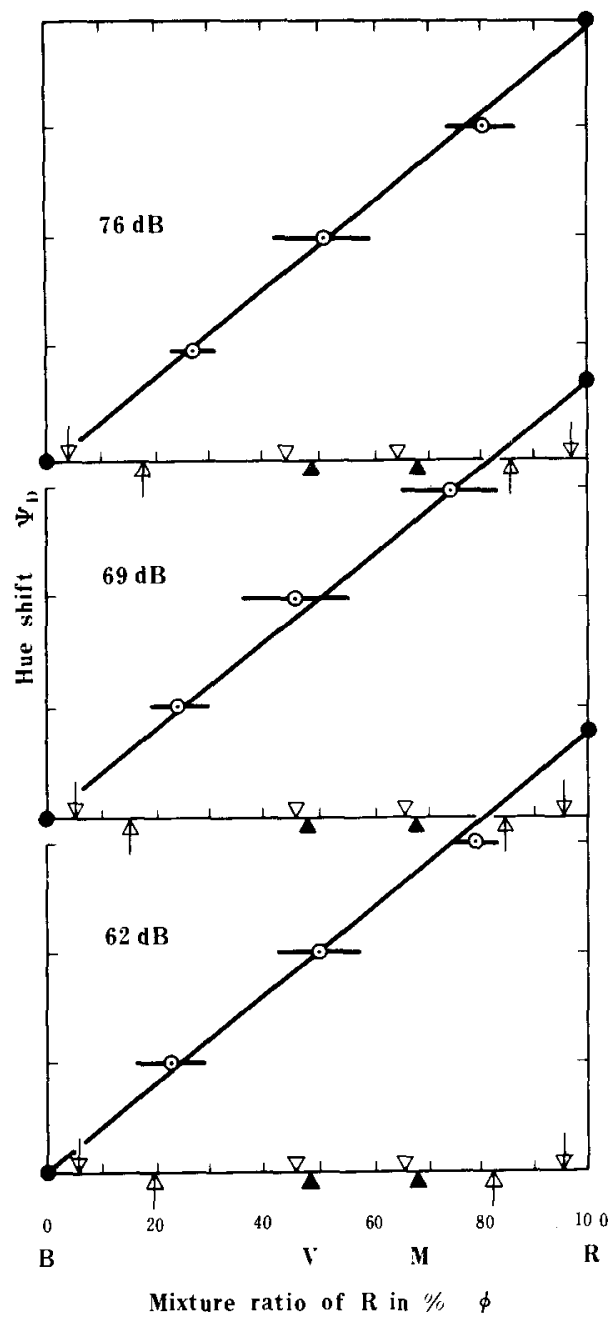

Figure 4. Equisections of hue shift between red, $R$, and blue, $B$ (Experiment 2.1). Horizontal bars represent standard deviation of settings. Arrows indicate absolute thresholds for $R$ and $B$ by ascending (arrow pointing up) and descending series (arrow pointing down) (Experiment 2.2). Filled triangles represent theoretical position of magenta, $M$, and violet, $V$, of $100 \% P C$; unfilled triangles, the results of matching by $R$ and $B$ (Experiment 2.3).

in Aperture 3 at the center, whereas $\mathrm{R}$ and $\mathrm{B}$ were mixed once in Aperture 2 and once in Aperture 4. Means of 30 matchings (two repetitions by 15 subjects) are given by the unfilled triangles in Figure 4. Inter- and intraindividual standard deviations of the settings were extremely small in this case. For some unknown reason, however, the means were systematically smaller, though only slightly, than the expected values.

\section{Experiments 2.4, 2.5}

Magnitude estimations by the ordinary procedure (Experiment 2.4) and by the method of combining magnitude production and estimation (Experiment 2.5) were conducted with the two component hues, redness and blueness, in a violet or magenta color. In both cases, the number to be assigned to the first stimulus, the modulus, was chosen by the subject, and the instructions emphasized that what was to be judged was not the depth or saturation of either violet or magenta itself but the degree of redness $\left(R^{\prime}\right)$ or blueness $\left(B^{\prime}\right)$ in the intermediate color. A series of five stimuli was presented, one by one, in Aperture 3 in Experiment 2.4, and the stimulus in Aperture 3 was set by the subject at 9-25 levels of $\phi$ in Experiment 2.5.

In the previous study (Indow \& S. S. Stevens, 1966, Experiment 4.1), direct judgment of yellowness ( $\left.Y^{\prime}\right)$ in the hue shifts R-Y and also Y-G was found to be extremely difficult for some subjects, and one subject had to be discarded in the magnitude estimation of $Y^{\prime}$ in R-Y and two subjects in Y-G. There was no problem in the direct judgments of redness $\left(R^{\prime}\right)$ in the former and of greenness $\left(G^{\prime}\right)$ in the latter. In the present case, all subjects could make the required judgment with $R^{\prime}$ and $\mathbf{B}^{\prime}$.

As the numbers of subjects were 6 and 15 in Experiments 2.4 and 2.5 , respectively, and two repetitions were made in each, 12 and 30 individual sets of data were obtained for each of $\mathrm{R}^{\prime}$ and $\mathrm{B}^{\prime}$. For each of these sets, a straight line was fitted in log-log coordinates to give the exponent, $\beta$, of the power function between $\psi_{M}$ and $\phi$, where $\psi_{M}$ represents the number assigned to $\mathrm{R}^{\prime}$ or $\mathrm{B}^{\prime}$, and $\phi$ represents the mixing ratio of $\mathrm{R}$ or $B$, respectively. Because each subject, $i$, made two repetitions, two values of $\beta$ were obtained and the mean was defined as $\beta_{\mathrm{i}}$ for the component hue. The standard deviation of the two values was called intrasubject, $S_{i}$. In Table 4 are given the grand means over the subjects of $\beta_{\mathrm{i}}$ and $\mathrm{SD}_{\mathrm{i}}$ thus defined, and also the standard deviations of $\beta_{\mathrm{i}}$ (intersubject SD). On the average, the exponents, $\beta$, were about the same in value between Experiments 2.4 and 2.5 , and the averages of the two are given in the last column. As a measure of the fit of the power function, the correlation, $\mathrm{r}$, between $\log \psi_{\mathrm{M}}$ and $\log \phi$ was used, as in Experiment 1.3. The ranges of $r$ for all the individual fits are included in Table 4.

The results in Table 4 may be compared with those for the hue shifts reported in Indow \& S.S. Stevens (1966). In the hue shifts between R and Y, R-Y, as well as between $Y$ and $G, Y-G$, both being at $81 \mathrm{~dB}$, the two component hues in each hue shift had about the same exponent values. Furthermore, the exponent for redness, $R^{\prime}$, in $R-Y$ was of the same order of magnitude as the exponent for saturation of $R$ at the same luminance, and the same was true for the exponent for greenness, $\mathrm{G}^{\prime}$, in $\mathrm{Y}-\mathrm{G}$ and the exponent for saturation of $G$. However, the exponents for yellowness, $Y^{\prime}$, in R-Y and also in Y-G were much smaller than the exponent for saturation of $Y$ at the same luminance. In the hue shift between $R$ and $B$ of the present study, the hue component, $\mathbf{B}^{\prime}$, tends to have larger exponents than the other hue component, $\mathrm{R}^{\prime}$, but this difference is due mainly to magnitude production and estimation 
Table 4

Exponents and Goodness of Fit for Magnitude Estimation (Experiment 2.4) and Magnitude Production and Estimation (Experiment 2.5) of Hue Shift

\begin{tabular}{|c|c|c|c|c|c|c|c|c|c|c|}
\hline \multirow[b]{2}{*}{ Hue } & \multirow[b]{2}{*}{ Luminance* } & \multicolumn{4}{|c|}{ Experiment 2.4} & \multicolumn{4}{|c|}{ Experiment 2.5} & \multirow[b]{2}{*}{$\begin{array}{c}\text { Meant } \\
\beta \\
\end{array}$} \\
\hline & & Mean & $\begin{array}{l}\text { Inter- } \\
\text { subject } \\
\text { SD }\end{array}$ & $\begin{array}{c}\text { Intra- } \\
\text { subject } \\
\text { SD }\end{array}$ & $\begin{array}{c}\mathrm{r}^{* *} \\
\text { Range }\end{array}$ & Mean & $\begin{array}{c}\text { Inter- } \\
\text { subject } \\
\text { SD }\end{array}$ & $\begin{array}{l}\text { Intra- } \\
\text { subject } \\
\text { SD }\end{array}$ & $\begin{array}{c}\mathrm{r} * * \\
\text { Range }\end{array}$ & \\
\hline $\mathbf{R}^{\prime}$ & $\begin{array}{l}76 \\
69 \\
62\end{array}$ & $\begin{array}{l}2.97 \\
2.57 \\
2.52\end{array}$ & $\begin{array}{r}1.08 \\
1.27 \\
.92\end{array}$ & $\begin{array}{l}.15 \\
.18 \\
.21\end{array}$ & $\begin{array}{l}.96-.99 \\
.94-.99 \\
.91-.99\end{array}$ & $\begin{array}{l}2.61 \\
2.21 \\
1.93\end{array}$ & $\begin{array}{r}1.12 \\
.86 \\
.91\end{array}$ & $\begin{array}{l}.34 \\
.32 \\
.48\end{array}$ & $\begin{array}{r}.74-.98 \\
.56-.99 \\
.73-.99\end{array}$ & $\begin{array}{l}2.79 \\
2.39 \\
2.23\end{array}$ \\
\hline $\mathbf{B}^{\prime}$ & $\begin{array}{l}76 \\
69 \\
62\end{array}$ & $\begin{array}{l}2.50 \\
2.72 \\
2.46\end{array}$ & $\begin{array}{r}.89 \\
1.11 \\
.94\end{array}$ & $\begin{array}{l}.12 \\
.17 \\
.24\end{array}$ & $\begin{array}{r}.95-.99 \\
.94-.98 \\
.95-.98\end{array}$ & $\begin{array}{l}3.30 \\
3.29 \\
2.06\end{array}$ & $\begin{array}{l}1.78 \\
1.69 \\
1.40\end{array}$ & $\begin{array}{l}.34 \\
.38 \\
.39\end{array}$ & $\begin{array}{r}.62-.99 \\
.87-.99 \\
.78-.99\end{array}$ & $\begin{array}{l}2.90 \\
3.01 \\
2.26\end{array}$ \\
\hline
\end{tabular}

*dB re $10^{-10} \mathrm{~L} . \quad$ ** Correlation between $\log \psi_{M}(\phi)$ and $\log \phi$. tMean of exponents in the two experiments.

(Experiment 2.5). According to Tables 3 and 4, the exponents for $\mathrm{R}^{\prime}$ in the hue shift and the exponents for saturation of $\mathrm{R}$ at the same luminances are of the same order of magnitude, whereas the exponents for $B^{\prime}$ tend to be larger than the exponents for saturation of $B$ at the same luminances, in both Experiment 2.4 and Experiment 2.5 .

\section{Experiment 2.6}

A mixture of $\mathrm{R}$ and $\mathrm{B}$ was presented in Aperture 3 as a standard and colors $R$ and $B$ in the two adjacent apertures were adjustable. All three were of the same luminance. The subject's task was to match the saturation of $R$ with the redness $\left(R^{\prime}\right)$ and the saturation of $B$ with the blueness $\left(B^{\prime}\right)$ of the standard. The mixture ratio, $\phi$, of the standard was varied in five ways, and matchings were repeated twice by each of 10 subjects. It was emphasized in the instructions that what was to be matched was not the saturation of violet or magenta itself, but the relative amount of the two components, $\mathrm{R}^{\prime}$ and $\mathrm{B}^{\prime}$.

Should the procedure of this experiment be regarded as a heterochromatic matching as in Experiment 1.4, the $\mathrm{Pc}$ of the adjusted color, $\mathrm{R}$ or $\mathrm{B}$, corresponds to $\phi_{\mathrm{j}}$ in Equation 1 and the mixture level of $\mathrm{R}$ or $\mathrm{B}$ in the standard color corresponds to $\phi_{\mathrm{k}}$. It is expected, by the same logic stated in the section of Experiment 1.4, that $\log \phi_{\mathrm{j}}$ is a linear function of $\log \phi_{\mathrm{k}}$ with a slope of $b_{j k}$ and the value of the regression coefficient of saturation upon hue components should be as follows:

$$
\mathrm{b}_{\mathrm{R} \mathrm{R}^{\prime}}=\frac{\beta_{\mathrm{R}^{\prime}}}{\beta_{\mathrm{R}}}, \quad \mathrm{b}_{\mathrm{BB}^{\prime}}=\frac{\beta_{\mathrm{B}^{\prime}}}{\beta_{\mathrm{B}}} .
$$

The former part of the expectation, linearity between $\phi_{j}$ and $\phi_{\mathrm{k}}$ on $\log -\log$ coordinates, was supported, as shown in Figure 5 , in which the grand mean of $\mathrm{Pc}$ for $\mathrm{R}$ or $\mathrm{B}$ is plotted against the mixture ratio of $\mathrm{R}$ or $\mathrm{B}$. There was no consistent difference among the results of these luminance levels, and all were fitted by a single straight line.
The second part of the expectation concerning the slope, $b_{R R^{\prime}}$ or $b_{B_{B}}$, was not completely supported. For $b_{R R^{\prime}}=1.1$, ratios of $\beta_{R}$, to $\beta_{R}$ are 1.0-1.6, 0.81.4 , and 1.0-1.3 at 76,69 , and $62 \mathrm{~dB}$, respectively. For $b_{B_{B}}=0.5$, ratios of $\beta_{B^{\prime}}$ to $\beta_{B}$ are 1.2-1.7, 1.5.1.9, and 1.3-1.5. The values of $\beta_{\mathrm{R}}$ and $\beta_{\mathrm{B}}$ are given in Table 3 (the third and last columns), and the values in the last column of Table 4 were used as $\beta_{\mathrm{R}^{\prime}}$ and $\beta_{\mathrm{B}^{\prime}}$.

The same experiment was conducted with $R^{\prime}$ and $Y^{\prime}$ in the hue shift R-Y and $Y^{\prime}$ and $G^{\prime}$ in the hue shift Y.G (Indow \& S. S. Stevens, 1966, Experiment 4.2). Agreement between $b$ and ratio of $\beta$ s was as follows.

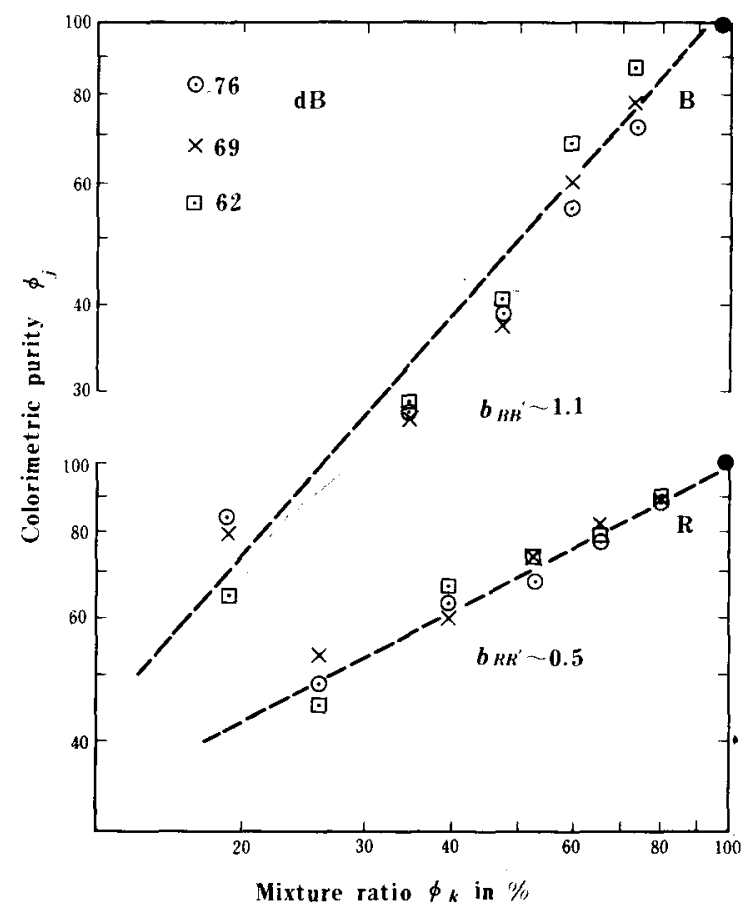

Figure 5. Matching of redness, $R^{\prime}$, or blueness, $B^{\prime}$, in a mixture of $R$ and $B$ (abscissa) with saturation of red, $R$, or blue, $B$ (ordinate), in log-log cordinates at three luminance levels (Experiment 2.6). 
Each figure in parenthesis represents expected values, i.e., the ratio of $\beta$ of magnitude estimation of the hue component to $\beta$ for magnitude estimation of the saturation. In R-Y, $b_{R_{R}}=0.9(1.11)$, and $b_{Y Y^{\prime}}=0.3(0.63)$, in $\mathrm{Y}-\mathrm{G}, \mathrm{b}_{\mathrm{YY}^{\prime}}=0.4(0.46)$, and $\mathrm{b}_{\mathrm{GG}^{\prime}}=0.9(0.90)$. At least in two cases, $b_{B B^{\prime}}$ in R-B of the present study and $b_{Y Y^{\prime}}$ in $Y-G$ of the previous study, the two experiments of direct judgment concerning hue component in an intermediate color (magnitude estimation and matching with saturation) failed to give a consistent result. Because both are concerned with perceptual hue component (not cancellation, for example), the failure cannot be ascribed to the so-called veiling effect (Krantz, 1975; Raaijmakers \& de Weert, 1975).

\section{GENERAL DISCUSSION}

For aperture colors in a dark surround, saturation behaves exactly the same as a function of colorimetric purity, $\phi$, no matter what the colors are: primary hues ( $R, Y, G$, and B), intermediate hues (YR and GY), and nonspectral intermediate hues (M and $\mathrm{V}$ ). Of the difference scale, $\psi_{\mathrm{D}}$, obtained by equisection, we cannot go beyond the following regularity, because $\psi_{D}$ for different hues is defined in terms of different units. Always, $\psi_{\mathrm{D}}$ is a linear to $\phi$ and intersects the abscissa at a point between $\phi=0$ and the absolute threshold. Of the magnitude scale, $\psi_{\mathrm{M}}$, obtained by magnitude estimation, etc., we can make better use of the finding that $\psi_{M}$ is always a power function of $\phi$.

Figure 6 summarizes the absolute thresholds and the overall results concerning the magnitude scale, $\psi_{M}$, for saturation at four luminance levels. Some values for spectral colors were obtained by interpolation from Figure 11 in the preceding study. For YR, Y, GY, and $G$, it was impossible to extrapolate up to the level of $62 \mathrm{~dB}$, hence there are no dotted lines on the right half of Figure 6. On the other hand, the results for $81 \mathrm{~dB}$ were obtainable only for spectral colors between $\mathrm{R}$ and $\mathrm{B}$. The lowest Plot I shows the absolute thresholds, $\phi_{0}$, for saturation in terms of colorimetric purity, Pc. As stated in the section of Experiment 1.2, $\phi_{0}$ for $\mathbf{R}$ and $\mathrm{B}$ obtained in the present experiments did not coincide in value with the corresponding $\phi_{0}$ in the previous study. Hence, all the values of $\phi_{0}$ in the present study were multiplied arbitrarily by 0.45 so as to be comparable with the results of the previous study.

The Plots II, III, and IV show the exponents, $\beta_{\mathrm{j}}$ of $\psi_{\mathrm{M}}$, by magnitude production and estimation (Experiment 1.3) and by heterochromatic matching (Experiment 1.4). Experiment 1.4 gives only relative values of exponents, $\beta_{\mathrm{j}}^{\prime}$. Those values were converted so as to coincide with $\beta_{\mathrm{j}}$ in Experiment 1.3 as closely as possible, and they are plotted in III (Experiment 1.4A). In Plot IV (Experiment 1.4B), the relative exponents, $\beta_{j}^{\prime}$, were converted so as to be comparable to the exponents, $\beta$, in the preceding experiment on the right
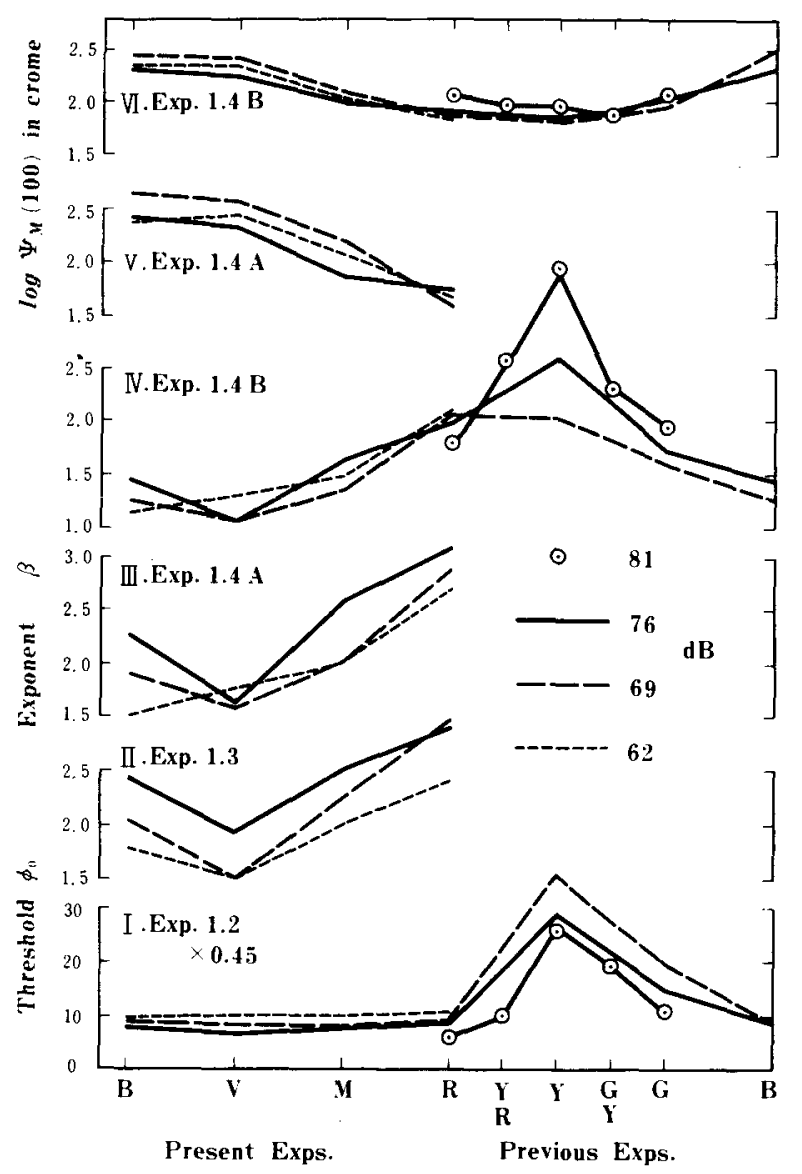

Fïgure 6. Summary of change of absolute threshold, exponent, and saturation of $100 \%$ Pc color as a function of hue varying from red, $R$, to blue, $B$, through yellow, $Y$, green, $G$, and nonspectral hues, magenta, $M$, and violet, $V$. Results in the spectrum were taken from a previous experiment (Indow \& S. S. Stevens, 1966). A and $B$ refer to alternative conversions of the same data (see text).

half of Figure 6. The values of $\beta_{R}$ for red given in the upper plot of Figure 3 are those used in this conversion.

In order to have the values of $\max \psi_{\mathrm{M}}(100)$ at $\mathrm{Pc}$ of $100 \%$, we need the values of the exponent, $\beta_{\mathrm{j}}$, and the values of the other parameter of the power function, $\alpha_{j}$. The relative values of $\alpha_{j}$ were obtained in Experiment $1.4\left(\alpha_{j}^{\prime}\right)$. In order to convert these relative values to absolute ones, we have to know the absolute value of at least one $\alpha$ The values of $\alpha_{R}$ and $\alpha_{B}$ in Figure 11 of the previous study were used for this purpose. S. S. Stevens called this scale $\psi_{\mathrm{M}}$ crome. In Figure 6, Plot V represents $\log \psi_{M}(100)$, obtained by the use of $\beta_{j}$ in Plot III (Experiment 1.4A) and Plot IV represents $\log \psi_{\mathrm{M}}(100)$, obtained by the use of $\beta_{\mathrm{j}}$ in Plot IV (Experiment 1.4B). The values in Plot VI can be linked with $\psi_{M}(100)$ in the preceding experiment in the right half of Figure 6. Because the ordinate of VI is in log units, the difference of maximum saturation between $R$ and $B$ may look small. However, the difference is very large in terms of 
$\psi_{\mathrm{M}}$. As seen in Figure 3, the consistency of heterochromatic matching in Experiment 1.4 is not quite satisfactory with regard to $\beta_{j}$. Hence, the absolute values of $\psi_{\mathrm{M}}(100)$ in Plot VI should not be taken too seriously.

Figure 6 exhibits the general pattern of change in the parameters along the spectral locus and the nonspectral region, even though their absolute values are uncertain. Threshold $\phi_{0}$ is almost constant and relatively unaffected by luminance in the nonspectral regions, whereas, $\phi_{0}$ changes as a function of hue as well as of luminance in the middle part of the spectral locus. Exponent $\beta$ seems to be $\mathrm{m}$ nimal at the middle of the nonspectral region (V) and maximal at about the middle of the spectral region (Y) and to be affected by luminance in different ways according to hue. Guirao and Mattiello (1974) gave a similar pattern of $\beta$ for colors which were more of object color mode in appearance, and the minimum $\beta$ was at $B$ in their results rather than at $V$. They did not give the luminance level of each hue, and it is likely that this difference is due to a difference in luminance. The saturation of the purest color, $\psi_{M}(100)$, reaches a maximum at $B$ and a minimum in the region from $Y R$ to $G Y$ and again varies in a complicated way according to luminance.

As to continuous change of hue, only shifts between two adjacent primary hues were dealt with in the present and previous experiments, for example, the shift between $R$ and $Y$. All colors in between have redness, $R^{\prime}$, and yellowness, $Y^{\prime}$, to different degrees, and these hue components were scaled by direct judgments. The shifts, R-Y, Y.G, and R-B, are cases in which the concomitant change of $P c$ is minimal. That does not necessarily mean that no change of saturation is involved, because saturation of intermediate colors of $100 \% \mathrm{Pc}$ may change as a function of position between the two primary hues. Plot VI of Figure 6 suggests that the change may be considerable in R-B through $M$ and $V$. Nevertheless, change of hue component was always found functionally analogous to saturation, shift from an achromatic color to a color of $100 \% \mathrm{Pc}$. Of all principal hue components, $R^{\prime}, Y^{\prime}, G^{\prime}$, and $B^{\prime}$, the difference scale, $\psi_{\mathrm{D}}$, is linearly related to mixture ratio of the hue, $\phi$, from $0 \%$ to $100 \%$ and the magnitude scale, $\psi_{M}$, is a power function of $\phi$. The shift, R-B, through the nonspectral region behaves in the same fashion as hue shifts along the spectral locus, R-Y and Y-G. It seems unnecessary, therefore, to assume an additional primary hue in the nonspectral region. The Munsell color system counts purple as a primary hue. This difference may be due to the fact that the Munsell system is concerned with object colors and the present experiment is concerned with aperture colors in a dark surround. Rather, purple (5P) has been used as a reference axis in the Munsell system to fill in the large gap between red and blue and is not primary in the sense that the other four primary hues are.

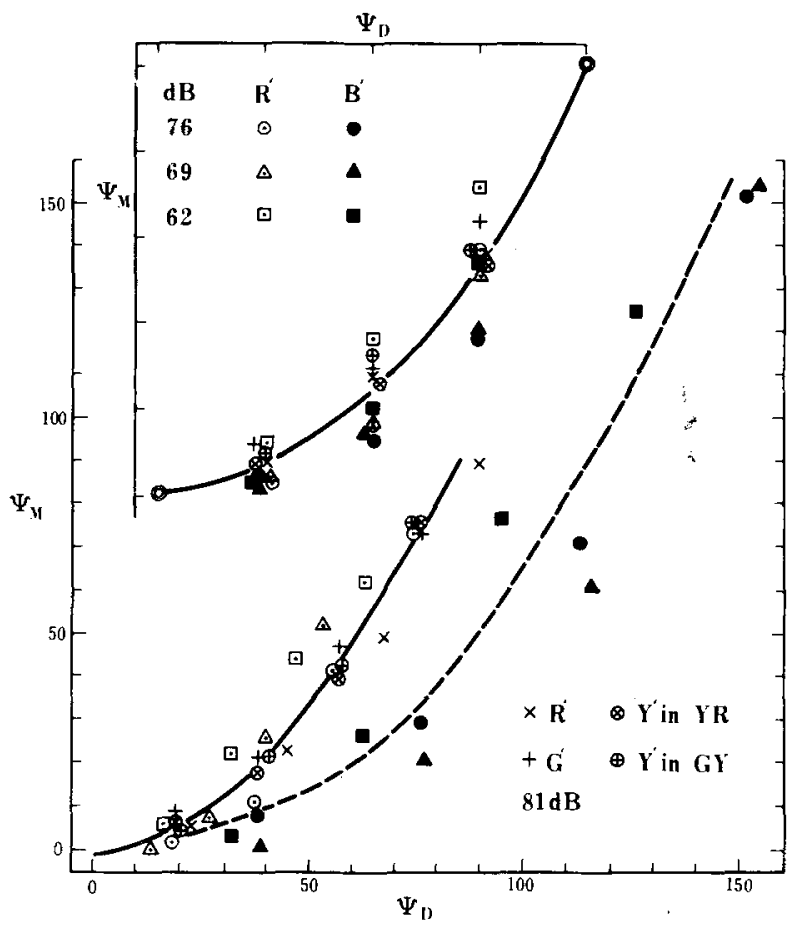

Figure 7. Nonlinearity between scale $\psi_{D}$ by equisection and scale $\psi_{M}$ by magnitude estimate and also magnitude production and estimation. $R^{\prime}, B^{\prime}$ at three luminance levels are from the present experiment; $R^{\prime}, Y^{\prime}$ in $Y R$ and $G^{\prime}, Y^{\prime}$ in $G Y$ are from a previous experiment (Indow \& S. S. Stevens, 1966). Dashed curve represents separate trend for $B^{\prime}$ (see text).

Since the magnitude scale, $\psi_{\mathrm{M}}$, is a power function of the stimulus value, $\phi$, with exponent $\beta>1$ and the difference scale, $\psi_{\mathrm{D}}$, is almost linear with $\phi, \psi_{\mathrm{M}}$ is always convex downward when plotted against $\psi_{\mathrm{D}}$. This is true both for the saturation of hues and for the hue shifts. The degree of convexity depends upon the value of $\beta$ and the range, $\left[\psi\left(\phi_{\mathrm{L}}\right), \psi\left(\phi_{\mathrm{U}}\right)\right]$, which is equisected to define $\psi_{\mathrm{D}}$. As to saturation of the four primary hues, $\psi_{M}$ was plotted against $\psi_{D}$ in two ways according to the definition of the range (Indow, 1974b, Figure 6). For the hue shifts, the corresponding plots are shown in Figure 7, and the hue shifts in the previous experiment, $R-Y$ and $Y-G$, are included in addition to the hue shift, R-B, in the present experiment. In the upper plot, both ranges, $\left[\psi_{D}(0), \psi_{D}(100)\right]$ and $\left[\psi_{M}(10), \psi_{M}(100)\right]$, were defined as $(0,100)$ for all cases. In Figure 4, the straight lines pass through points, $\phi=0$, and $\phi=100$, and not the thresholds, $\phi_{0}$, on both sides. This is the reason for having defined $\psi_{\mathrm{D}}(0)$ and $\psi_{\mathrm{D}}(100)$, not $\psi_{\mathrm{D}}\left(\phi_{0}\right)$, equal to 0 and 100 , respectively. The values of $\psi_{D}$ for $R^{\prime}$ and $B^{\prime}$ were read from Figure 4, and the values of $\beta$ in the last column of Table 4 were used to define $\psi_{M}$.

In the upper plot of Figure 7, points representing $\mathbf{B}^{\prime}$, especially of lower luminance levels (filled circles and triangles) deviate from the general trend. The anom- 
alous behavior of $\mathrm{B}$ was also noticeable, though slighter, in the same plot of $\psi_{\mathrm{D}}$ and $\psi_{\mathrm{M}}$ for saturation in Figure 6 of the previous article (Indow, 1974b). In the lower plot of Figure 7, the ranges, $\left[\psi_{\mathrm{D}}(0),{ }_{\mathrm{D}}(100)\right]$ and $\left[\psi_{M}(0), M_{M}(100)\right]$, were defined as $\left[0, \psi_{M}(100)\right]$ for all cases, where the values of $\psi_{\mathrm{M}}(100)$ were taken from Plot VI in Figure 6. In this plot, points representing $R^{\prime}$ in $R-B$ and $R \cdot Y, Y^{\prime}$ in, $R-Y$ and $Y-G$, and $G^{\prime}$ in $Y \cdot G$ are described more closely by a single curve, convex downward (solid curve). On the other hand, the relation between $\psi_{D}$ and $\psi_{M}$ for $B^{\prime}$ in R-B is clearly different from the general irend at all three luminance levels (dashed curve for filled circles. triangles, and squares).

The same kind of nonlinearity between $\psi_{D}$ and $\psi_{M}$ has been amply shown in other sense modalities. In a plot similar to Figure 7, it was shown that brightness of object color, loudness of tone, and sweetness form a single curve, whereas numerosity of randomly scattered dots and hardness of pencil lead form another curve (Indow \& Ida, 1977). Both are convex downward, and the latter is slightly less curved than the former. The difference may be due to the fact that the widest possible range of $\phi$ for the modality is dealt with in each of the former groups, which cannot be claimed in the latter two examples. In the present study, it can be said that the widest possible range is covered in each hue shift. It is of interest, therefore, that a single general trend has been observed for $R^{\prime}, Y^{\prime}$, and $G^{\prime}$ with one exception ( $\mathrm{B}^{\prime}$ ).

Evidently, the difference between $\psi_{\mathrm{M}}$ and $\psi_{\mathrm{D}}$ consists in the fact that the former is based upon "ratio" judgments and the latter upon "difference" judgments. According to Krantz (1974), "One thing is obvious: the 'ratio' and 'difference' judgments are not ratios and differences of a common psychophysical scale" (p. 195). As the nonlinearity between $\psi_{\mathrm{M}}$ and $\psi_{\mathrm{D}}$ is a universal phenomenon and quantitative agreement is observed within some sets of modalities, the origin of the nonlinearity must lie in the nature of information processing, through which the two kinds of quantitative judgments are generated, rather than in respective sensory processes.

\section{REFERENCES}

Guirao, M., \& DeMatiello, M. L. F. Saturation scales for surface colors. Vision Research, 1974, 14, 487-493.
INDow, T. Saturation scales for red. Vision Research, 1967, 7, 481-495.

Inoow, T. Colour atlases and colour scaling. Association Internationale de la Couleur, Coulour '73. Adam Hilger, 1974, 137-152. (a)

INDow, T. Scaling of saturation and hue shift: Summary of results and implication. In H. R. Moskowitz, B. Scharf, \& J. C. Stevens (Eds.), Sensation and measurement-Papers in honor of S. S. Stevens. Dordrecht, Holland: Reidel, 1974. Pp. 351-362. (b)

Indow, T.. \& IDA, M. Scaling of dot numerosity. Perception \& Psychophysics, 1977, 22, 265-276.

InDow, T., \& STEvens, S. S. Scaling of saturation and hue. Perception \& Psychophysics, 1966, 1, 253-271.

Krantz, D. H. Measurement theory and qualitative laws in psychophysics. In D. H. Krantz, R. C. Atkinson, R. D. Luce, \& P. Suppes (Eds.), Contemporary developments in mathematical psychology (Vol. II), Measurement, psychophysics, and neural information processing, San Francisco: Freeman, 1974.

Krantz. D. H. Color measurement and color theory: II. Opponent-colors theory. Journal of Mathematical Psychology, 1975, 12, 304-327.

Markes, L. E., \& Stevens, J. C. Individual brightness function. Perception \& Psychophysics, 1966, 1, 17-24.

Mosteller, F. Remarks on the method of paired comparisons: I. The least squared solution assuming equal standard deviations and equal correlations. Psychometrika, 1951, 16, 3-9.

RaAijmakers, J. G. W., \& DE WeERT, C. M. M. Linear and nonlinear opponent color coding. Perception \& Psychophysics, $1975,18,474-478$.

Stevens, J. C., \& Guirao, M. Individual loudness functions. Journal of the Acoustical Society of America, 1964, 36, 2210-2213.

Stevens, S. S. Decibels of light and sound. Physics Today, 1955, 8. $12-17$.

Stevens, S. S. The psychophysics of sensory function. In W. A. Rosenblith (Ed.), Sensory communication. Cambridge, Massachusetts: MIT Press, 1961. Pp. 1-33.

Stevens, S. S. In G. Stevens (Ed.), Psychophysics: Introduction to its perceptual. neural, and social prospects. New York: Wiley, 1975.

\section{NOTE}

1. The calibration of the apparatus, as noted by Indew (1967), was difficult and potentially biased both in the region of high mixture ratios above $90 \%$ and in the region of low mixture ratios below $10 \%$, due to internal reflection within the prism. These unreliable mixture ratios were used only in Experiments 1.2 and 2.2.
(Received for publication August 11, 1977; revision accepted April 11, 1978.) 